\title{
Evaluating the Degree of Macrodispersion of Carbon Nanotubes using UV-VIS-NIR Absorption Spectroscopy
}

\author{
Ki Kang Kim ${ }^{1}$, Soo Min Kim ${ }^{1}$, Yan Cui ${ }^{1}$, Mun Seok Jeong ${ }^{2}$, Jong Hun Han ${ }^{3}$, Young Chul Choi ${ }^{4}$, \\ Kay Hyeok $\mathrm{An}^{5}$, Kyunghui $\mathrm{Oh}^{6}$ and Young Hee Lee,
}

\author{
${ }^{1}$ BK21 Physics Division, Center for Nanotubes and Nanostructured Composites, Sungkyunkwan Advanced Institute of Nanotechnology, \\ Sungkyunkwan University, Suwon 440-746, Republic of Korea \\ ${ }^{2}$ Advanced Photonics Research Institute, Gwangju Institure of Science and Technology, Gwangju, 500-712, Republic of Korea \\ ${ }^{3}$ Energy and Nanomaterials Research Center, Korea Electronics Technology Institute, Seongnam, 463-816, Republic of Korea \\ ${ }^{4}$ R\&D Center, Hanwha Nanotech, 423-1 CheongCheon-dong, Bupyeong-gu, Incheon, 403-030, Republic of Korea \\ ${ }^{5}$ Material and Development Department Jeonju Machinery Research Center, Jeonju, 561-844, Republic of Korea \\ ${ }^{6}$ Korea Agency for Technology and Stardards, Gwacheon, 427-723, Republic of Korea \\ ‘e-mail: leeyoung@skku.edu \\ (Received February 14, 2009; Accepted March 16, 2009)
}

\begin{abstract}
We measured the degree of macrodispersion of the various single-walled carbon nanotubes (SWCNTs) and multi-walled carbon nanotubes (MWCNTs) using UV-VIS-NIR absorption spectroscopy. CNTs were dispersed with SDS of 2 wt \% in deionized water using the homogenizer and then were further centrifugated at $6000 \mathrm{~g}$ for $10 \mathrm{~min}$. The degree of macrodispersion, expressed by $D_{m}(\lambda)=A_{a}(\lambda) / A_{b}(\lambda)^{*} 100(\%)$, where $\lambda$ is the wavelength and $A_{a}(\lambda)$ and $A_{b}(\lambda)$ are the absorbance of the sample after and before centrifugation, respectively. In the case of MWCNTs, we evaluated the degree of macrodispersion by the average degree of macrodispersion $\left(\mathrm{D}_{\mathrm{m}}(\lambda)\right)$ between 1000 and $1200 \mathrm{~nm}$. The degree of macrodispersion of SWCNTs was evaluated at the wavelength in which van Hove singularity-related transition regions were excluded, i.e., the range was chosen between $\mathrm{E}_{11} \mathrm{~s}$ and $\mathrm{E}_{22} \mathrm{~s}$ peaks. We have estimated six samples with the same method. The standard deviation of each sample was lower than 5. Therefore, we presented a reliable evaluation method for the macrodispersion of CNTs for standardization.
\end{abstract}

Keywords : Carbon nanotube, Dispersion, UV-VIR-NIR spectroscopy, Standardization

\section{Introduction}

Since the discovery of carbon nanotubes (CNTs) in 1991 [1], CNTs have been developed for numerous applications including gas sensor [2], nano-composite [3], field-effect transistor [4], transparent conducting film [5], and cooler [6], due to their higher mechanical properties, thermal conductivity, tensile strength, electrical conductivity, and etc [7]. CNTs have been synthesized by several methods such as arc discharge [1], catalytic chemical vapor deposition (CVD) [8], high pressure carbon monoxide process (HIPCO) [9], CoMoCat process [10], and laser ablation [11] using various catalysts of $\mathrm{Fe}, \mathrm{Co}, \mathrm{Mo}$, $\mathrm{Ni}$, their mixtures, and so on. These CNTs exist in bundled form in a few micrometers long due to van der Waals interaction energy of about $1000 \mathrm{eV}$ [12]. The dispersion of the raw SWCNTs into individual nanotubes or smaller bundle-size nanotubes are frequently required to maximize or to retain their superb properties in the numerous applications.

The dispersion of CNTs can be classified into macrodispersion and nanodispersion, which is defined as a macro state dispersed in solvent with small-size bundles and that with individual SWCNT, respectively $[13,14]$. In order to compare the dispersibility of CNTs, several methods have been used, for example, UV-VIS-NIR spectroscopy [15], fluorescence spectroscopy [16], atomic force microscopy (AFM) [17], and transmission electron microscopy (TEM) [18]. However, AFM and TEM do not offer the reliable data because the sample should be prepared on the substrate in a small quantity. UV-VIS-NIR spectroscopy not only measures dispersed CNTs solution directly, but also provides the quantitative data.

In spite of much effort, the standardized evaluation method for the degree of dispersion of CNTs has not been established yet as a standard protocol. This makes researcher or engineer to be confused which has been one of the ambiguities and therefore delays industry applications of CNTs. For these reasons, the evaluation method of the degree of the dispersion of CNTs should be addressed in terms of standardization [19].

In this paper, we suggested the evaluation method of the degree of macrodispersion of CNTs for standardization. Numerous dispersion methods have been reported elsewhere. 
For example, the surfactants such as sodium dodecyl sulfate (SDS) and sodium dodecylbenzene sulfonate (NaDDBS) can be used for the dispersion of CNTs in solution phase [16]. Organic solvents such as 1,2-dichloroethane (DCE), N,Ndimethylformamide (DMF) and N-methyl-2-pyrrolidone (NMP) have been utilized for the dispersion [17,20]. Kim et al. showed the designed dispersants based on the monomer thiophene to disperse CNTs with long-term stability in organic solvent [18]. We have estimated the degree of the macrodisepersion of CNTs using UV-VIS-NIR spectroscopy. For the standardization, we need to fix several variables in advance such as the amount of dispersant, solvent and CNTs, the shape of the flask, the solution temperature, the homogenizer power and time, the centrifugation speed and time. From the Beer's law (Absorbance $=\varepsilon \imath \mathrm{c}$, where $\varepsilon$ is the extinction coefficient, $\mathrm{l}$ is the path length, $\mathrm{c}$ is the concentration of CNTs), the absorbance only depends on the concentration of CNT, if the path length and extinction coefficient were a constant. In the previous work, Jeong et al. showed that the extinction coefficient of single-walled carbon nanotubes (SWCNTs) with a mean diameter of 1.4 $\mathrm{nm}$ is about $30 \mathrm{~mL} \cdot \mathrm{mg}^{-1} \cdot \mathrm{cm}^{-1}$ with a constant extinction coefficient [21]. Accordingly, we assumed that the absorbance is only related to the CNT concentration directly. The absorbance of the CNT solution before and after centrifugation become defined as $A_{b}(\lambda)$ and $A_{a}(\lambda)$, respectively. The degree of the macrodiseprsion of CNTs has a relationship as follow;

$$
D_{M}(\lambda)=\frac{A_{a}(\lambda)}{A_{b}(\lambda)} \times 100(\%)
$$

where $\lambda$ is the wavelength, $A_{b}(\lambda)$ and $A_{a}(\lambda)$ are the absorbance before and after the centrifugation. At this moment, if any sedimentation of CNTs occurs on the bottom of the flask after homogenizing, this solution cannot be further used for standardization. We have examined the several samples including multi-walled carbon nanotubes (MWCNTs) and SWCNTs. The obtained standard deviation (SD) value of $\mathrm{D}_{\mathrm{m}}$ is lower than 5. In this paper, we have suggested a reliable evaluation method of the macrodispersion of CNTs for the standardization protocol using UV-VIS-NIR spectroscopy. This method is expected to represent the degree of CNT dispersion, which can be used as a protocol of measuring the degree of macrodispersion of CNTs.

\section{Experimental}

\subsection{Preparation of carbon nanotube and dispersion solution}

Fig. 1 shows the schematic diagram of the sample preparation. Three types of MWCNTs (CM95, CB03, and CB04) used in this study were provided from Hanwha Nanotech. They have a mean diameter of $15 \mathrm{~nm}$. In the case

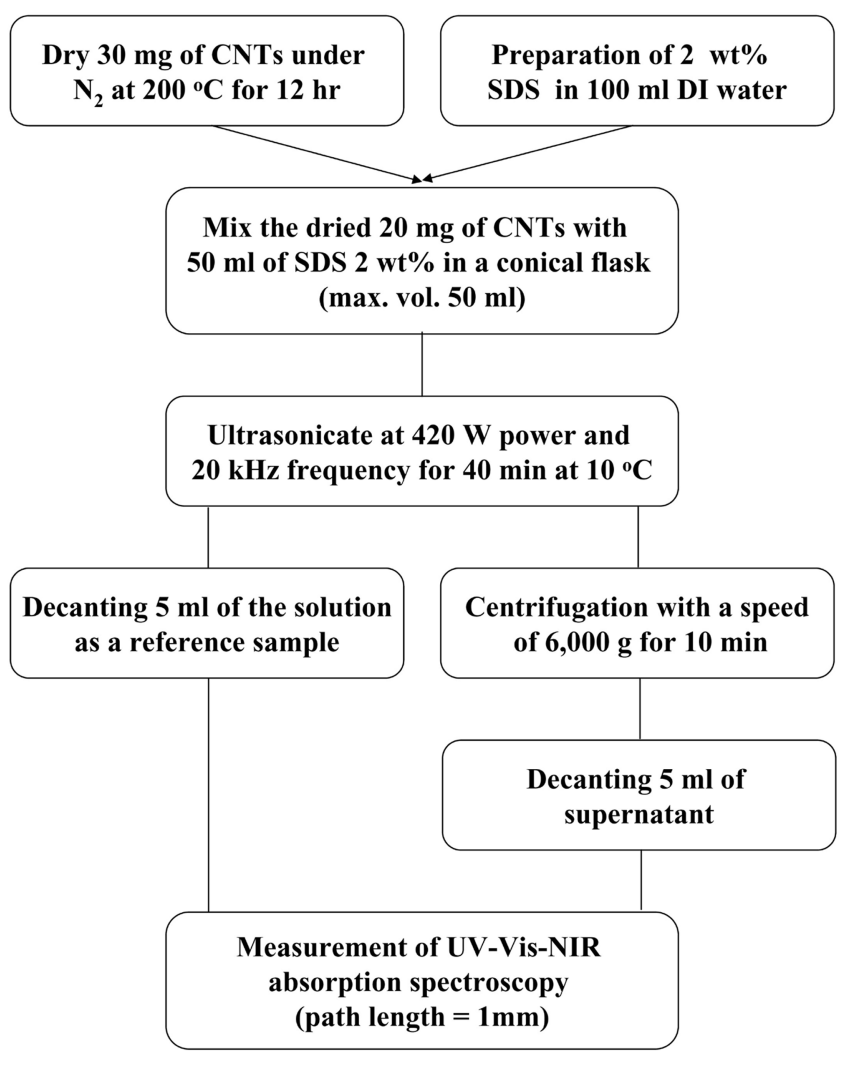

Fig. 1. The schematic diagram of the dispersion procedure.

of SWCNTs, the purified HIPCO ${ }^{\circledR}$ single-wall carbon nanotubes $(\mathrm{MD}=1 \mathrm{~nm})$, simply as a HIPCO, SWeNT ${ }^{\circledR} \mathrm{SG}$ 65 single-walled carbon nanotubes $(\mathrm{MD}=0.8 \mathrm{~nm})$, ASP$100 \mathrm{~F}$ sing-walled carbon nanotubes $(\mathrm{MD}=1.4 \mathrm{~nm})$ were purchased from Unidym, SouthWest Nanotechnologies, and Hanwha Nanotech, respectively. To exclude moisture or other effects, $30 \mathrm{mg}$ of all the samples were dried under nitrogen ambient at $200^{\circ} \mathrm{C}$ for $12 \mathrm{hr}$ in oven prior to dispersion.

We chose the dispersion method in aqueous solution with SDS as a surfactant, which is environment-friendly, cheep, and convenience. So, all CNT dispersion solutions were prepared using 2 wt \% SDS in $100 \mathrm{~mL}$ DI water.

\subsection{Dispersion procedure and centrifugation}

The $20 \mathrm{mg}$ of dried CNTs and $50 \mathrm{~mL}$ of SDS $2 \mathrm{wt} \%$ solution were placed in a $50 \mathrm{~mL}$ volumetric conical flask, which was used to spread out the ultrasound energy homogenously during the dispersion process. The dispersion was carried out by the homogenizer (ULH 700S, Taewon Science Company) at $420 \mathrm{~W}$ and $20 \mathrm{kHz}$ frequency at $10^{\circ} \mathrm{C}$ for $40 \mathrm{~min}$. The tip of homogenizer with a diameter of $7 \mathrm{~mm}$ was immersed $1 \mathrm{~cm}$ deep from the top of the solution surface in a conical flask. To maintain the temperature of the solution at $10^{\circ} \mathrm{C}$, the refrigerating bath circulator system 

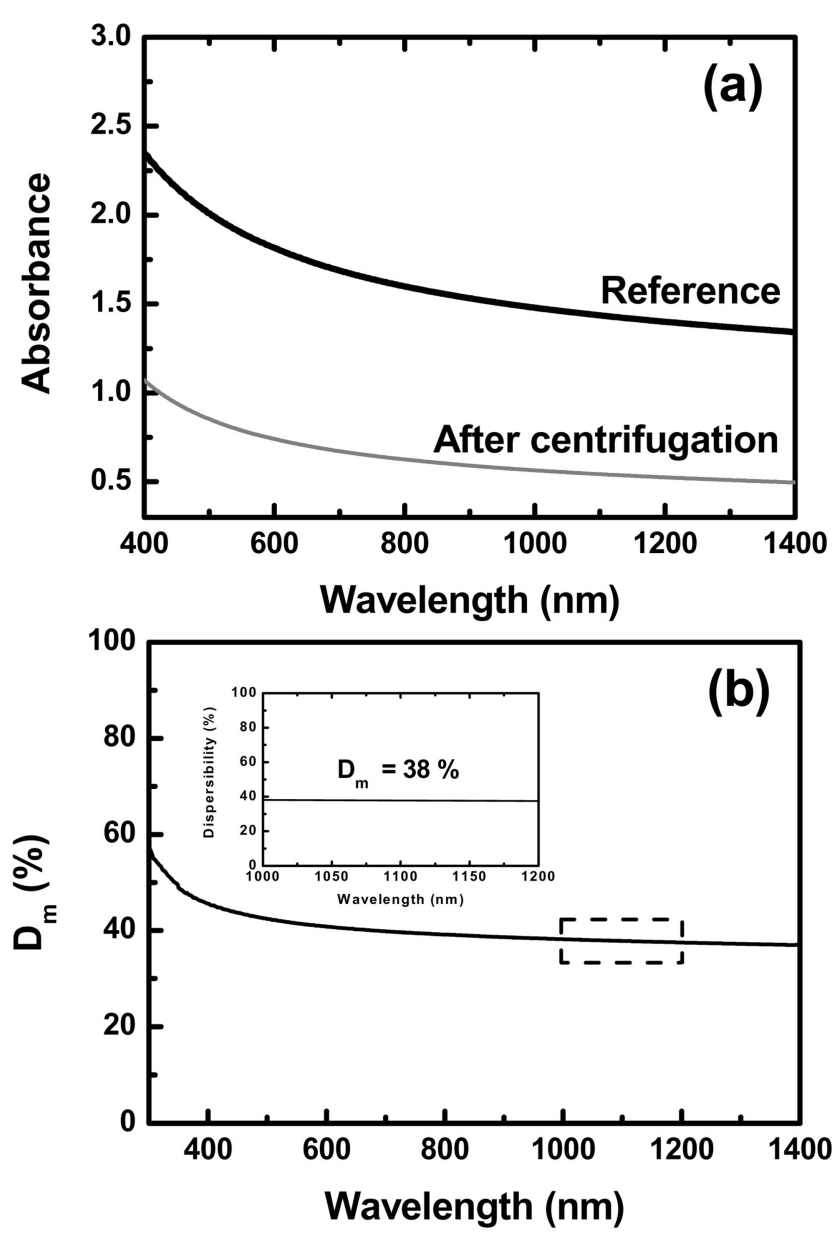

Fig. 2. (a) The UV-VIS-NIR absorption spectra of MWCNTs (CM95) before and after the centrifugation. (b) The degree of the macrodispersion of MWCNTs denoted as $D_{m}(\lambda)$ versus wavelength $(\mathrm{nm})$. The inset shows the $\mathrm{D}_{\mathrm{m}}(\lambda)$ between $1000 \mathrm{~nm}$ and $1200 \mathrm{~nm}$

(VTRC-620, JEIO Tech) was equipped in the outside of a conical flask.

After dispersion, $5 \mathrm{~mL}$ of the prepared CNT solution was decanted as a reference sample and followed by centrifugation (Mega 17R, Hanil Science Industrial Co. Ltd.) of $40 \mathrm{~mL}$ with a rotation speed of $6,000 \mathrm{~g}$ for $10 \mathrm{~min}$ to remove the severely entangled CNTs. The $5 \mathrm{~mL}$ of supernatant was also decanted from resulting solution.

\subsection{Measurement}

UV-VIS-NIR absorption spectroscopy (Cary 5000, Varian) was used to measure the absorbance of the reference samples and the centrifuged samples above. The path length of the spectroscopic quartz cell was $1 \mathrm{~mm}$. Identical experiments were taken three times per sample to obtain the average and standard deviation.

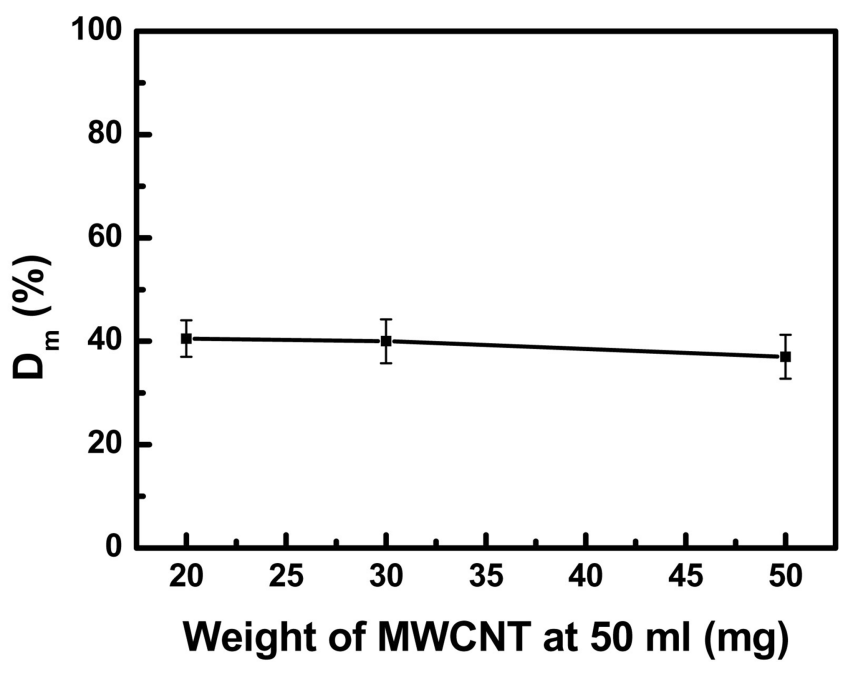

Fig. 3. The relation between $D_{m}$ and the concentration of MWNCT (CM95). The bar indicates the standard deviation (SD).

\section{Results and Discussion}

\subsection{Degree of macrodisperion of MWCNTs}

Fig. 2a shows the absorbance of the MWCNT (CM95) solution before and after the centrifugation. In general, the absorbance spectra of MWCNTs is only related to the $\pi$ plasmon peak around 4.0-4.25 eV. Accordingly, the absorbance of MWCNTs was increased toward the lower wavelength and was decreased due to the removal severely entangled CNTs after centrifugation. In the previous work, Geng et al. showed only the rope or individual MWCNTs after centrifugation using the scanning electron microscopy (SEM) [22]. The degree of macrodispersion of CNTs denoted as $D_{m}(\lambda)$ was dependent on the wavelength as shown in Fig. $2 b$. However, the inset in Fig. $2 b$ shows the uniform $D_{m}(\lambda)$ in the range between 1000 and $2000 \mathrm{~nm}$. Consequently, we selected this range to determine the $D_{m}$ as the following relationship to

$$
D_{m}=\frac{1}{201} \sum_{\lambda=1000}^{1200}\left(\frac{A_{a}(\lambda)}{A_{b}(\lambda)} \times 100\right)(\%) .
$$

As a result, we took $38 \%$ of $\mathrm{D}_{\mathrm{m}}$ of MWCNTs (CM95).

To investigate the dependence of $\mathrm{D}_{\mathrm{m}}$ on the concentration of MWCNTs, the weight was varied from $20 \mathrm{mg}$ to $50 \mathrm{mg}$ with the same amount of solvent. And we carried out the same experiment three times for each solution. In Fig. 3, for $20 \mathrm{mg}$ MWCNTs, the $\mathrm{D}_{\mathrm{m}}$ and their SD were $40 \%$ and 4 , respectively. The others were almost similar to the case of $20 \mathrm{mg}$ MWCNTs, but the $\mathrm{D}_{\mathrm{m}}$ of $50 \mathrm{mg}$ MWCNTs which was decreased by $3 \%$ compared to that of $20 \mathrm{mg}$ MWCNTs. This may be attributed from the limitation of the dispersibility of SDS. Therefore, we determined that the weight of CNTs was $20 \mathrm{mg}$ in $50 \mathrm{~mL}$ water for standardization. 

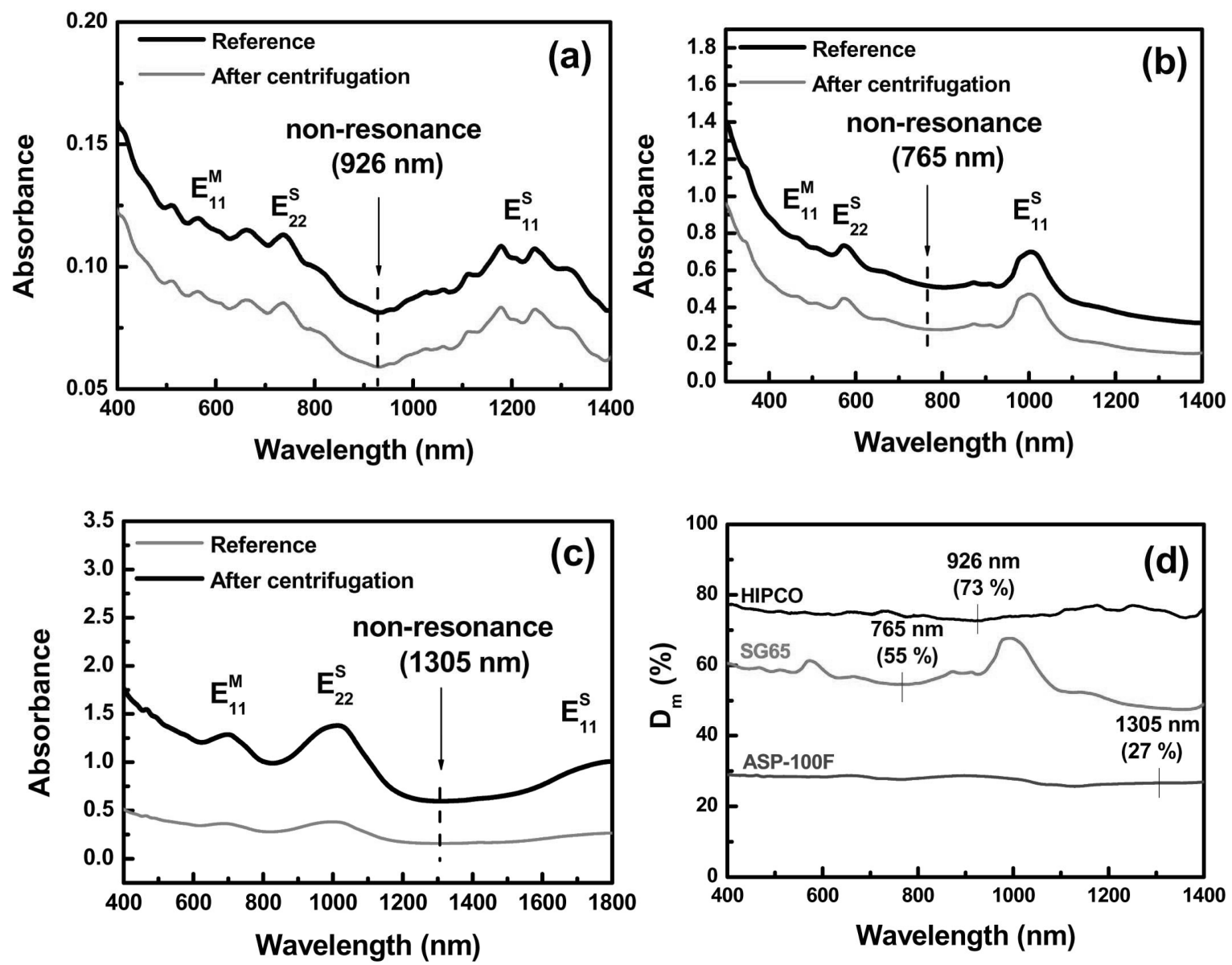

Fig. 4. The optical absorption spectra of (a) HIPCO SWCNTs, (b) SG65 SWCNTs, and (c) ASP-100F SWCNTs before and after the centrifugation. (d) The $\mathrm{D}_{\mathrm{m}}$ of each SWCNT. The arrow (or bar) indicates non-resonant wavelength of van Hove singularity-related transitions between $\mathrm{E}_{11} \mathrm{~S}$ and $\mathrm{E}_{22} \mathrm{~S}$.

\subsection{Degree of macrodisperion of SWCNTs}

The $\mathrm{D}_{\mathrm{m}}$ of the various kinds of SWCNTs was evaluated using same method with MWCNTs except averaging the $\mathrm{D}_{\mathrm{m}}(\lambda)$. Unlike MWCNTs in the optical spectra, the SWCNTs have the van Hove singularity (vHS)-related transition peaks such as $\mathrm{E}_{11} \mathrm{~s}$ and $\mathrm{E}_{22} \mathrm{~s}$ from the semiconducting SWCNT and $\mathrm{E}_{11}{ }^{\mathrm{M}}$ from the metallic SWCNT [23]. As shown in Fig. 4a-c, the vHS-related transition peaks are dependent on their diameter or optical band gap [23]. The $D_{m}(\lambda)$ of SWCNTs fluctuated with the wavelength, as shown in Fig. 4d. This originates from the different absorbance of $\mathrm{E}_{11} \mathrm{~s}, \mathrm{E}_{22} \mathrm{~s}$, and $\mathrm{E}_{11} \mathrm{M}$ peaks before and after the centrifugation. In the previous work, the absorbances of these peaks were stronger with increasing dispersibility of SWCNTs [15]. Consequently, the $\mathrm{D}_{\mathrm{m}}(\lambda)$ fluctuated among $\mathrm{E}_{11} \mathrm{~s}, \mathrm{E}_{22} \mathrm{~s}$, and $\mathrm{E}_{11}{ }^{\mathrm{M}}$ peaks. To obtain the reliable $\mathrm{D}_{\mathrm{m}}$ of SWNCTs, we selected the wavelength in which the vHS-related transition peaks were not visible between $\mathrm{E}_{11} \mathrm{~s}$ and $\mathrm{E}_{22} \mathrm{~s}$. For example, in the case of HIPCO SWCNTs, the wavelength of $926 \mathrm{~nm}$ was selected for the $D_{m}$. The wavelength of $765 \mathrm{~nm}$ and $1305 \mathrm{~nm}$ was chosen from SG65 and ASP-100F, respectively. In Fig. $4 \mathrm{~d}$, all the $\mathrm{D}_{\mathrm{m}}$ were flat near the selected wavelength.

In table 1 , the $\mathrm{D}_{\mathrm{m}}$ and $\mathrm{SD}$ of the various types of CNTs were summarized. Among the evaluated SWCNTs, the $\mathrm{D}_{\mathrm{m}}$ of HIPCO SWCNTs was the highest and the $\mathrm{D}_{\mathrm{m}}$ of CB03 MWCNTs was 59\%. The SDs of all the CNTs were lower than 5. This indicates that our suggested method is reliable for standardization.

\section{Conclusions}

Based on the Beer's law, we have suggested the evaluation method for the degree of macrodisepersion of CNTs to establish standardization protocol by comparing the absorbance of dispersed CNT solution before and after centrifugation in the UV-VIS-NIR absorption spectroscopy. The $\mathrm{D}_{\mathrm{m}}$ of MWCNTs and SWCNTs was 
Table 1. Degree of Macrodisperion of Various Types of CNTs

\begin{tabular}{cccccc}
\hline Model & Class & Wavelength & $\mathrm{D}_{\mathrm{m}}(\%)$ & SD & Company \\
\hline HIPCO & SWCNT & 926 & 78 & 5 & Unidym \\
SG65 & SWCNT & 765 & 54 & 2 & South West Nano Technologies \\
ASP-100F & SWCNT & 1305 & 29 & 2 & Hanwha Nanotech \\
CM95 & MWCNT & $1000 \sim 1200$ & 40 & 4 & Hanwha Nanotech \\
CB03 & MWCNT & $1000 \sim 1200$ & 59 & 1 & Hanwha Nanotech \\
CB04 & MWCNT & $1000 \sim 1200$ & 57 & 1 & Hanwha Nanotech \\
\hline
\end{tabular}

$$
D_{m}=\frac{1}{201} \sum_{\lambda=1000}^{1200}\left(\frac{A_{a}(\lambda)}{A_{b}(\lambda)} \times 100\right)(\%) \text { and } D_{m}=\frac{A_{a}(\lambda)}{A_{b}(\lambda)} \times 100(\%)
$$

at the specific wavelength in which the vHS-related transition peaks were not visible between $E_{11} \mathrm{~S}$ and $\mathrm{E}_{22} \mathrm{~S}$, respectively. The SD from our method was lower than 5 . We think that our method in this work provides an easy and simple way to determine the degree of dispersion and it may lead to establish the standardization protocol for the degree of the dispersion.

\section{Acknowledgment}

This work is financially supported by ITEP-Publication1002817.

\section{References}

[1] Iijima, S. Nature 1991, 354, 56.

[2] Kong, J.; Franklin, N. R.; Zhou, C.; Chapline, M. G.; Peng, S.; Cho, K.; Dai, H. Science 2000, 287, 622.

[3] a) Zhan. G.-D.; Kuntz. J. D.; Wan, J.; Mukherjee, A. K. Nature Mater. 2002, 2, 38. b) Kim S.J.; im J.S.; Kang P.H.; Kim T.; Lee Y.S.; Carbon Lett. 2008, 9, 294.

[4] Tans. S. J.; Verschueren, R. M.; Dekker, C. Nature 1998, $393,49$.

[5] Geng, H.-Z.; Kim. K. K.; So, K. P.; Lee, Y. S.; Chang, Y.; Lee, Y. H. J. Am. Chem. Soc. 2007, 129, 7758.

[6] Kordas, K.; Toth, G.; Moilanen, P.; Kumpumaki, M.; Vahakangas, J.; Uusimaki, A.; Vajtai, R.; Ajayan, P. M. Appl. Phys. Lett. 2007, 90, 123105.

[7] a) Baughman, R. H.; Zakhidov, A. A.; de Heer, W. A. Science 2002, 297, 787. b) Sharon M.; Rusop M.; Soga T.; Afre R.A. Carbon Lett. 2008, 9, 17. c) Sharon M.; Datta S.; Shah M.; Sharon M.W.; Soga T.; Afre R.A. Carbon Lett. 2007, 8, 184.

[8] Jeong, H. J.; Kim. K. K.; Jeong, S. Y.; Park, M. H.; Yang,
C. W.; Lee, Y. H. J. Phys. Chem. B 2004, 108, 17695.

[9] Bronikowski. M. J.; Willis, P. A.; Colbert, D. T.; Smith, K. A.; Smalley, R. E. J. Vac. Sci. Technol. A 2001, 19, 1800.

[10] Kitiyanan, B.; Alvarez, W. E.; Harwell, J. H.; Resasco, D. E. Chem. Phys. Lett. 2000, 317, 497.

[11] Park, Y. S.; Kim, K. S.; Jeong, H. J.; Kim, W. S.; Moon, J. M.; An, K. H.; Bae, D. J.; Lee, Y. S.; Park, G-S.; Lee, Y. H. Synth. Met. 2002, 126, 245.

[12] Sun, C.-H.; Yin, L.-C.; Li. F.; Lu, G.-Q.; Cheng, H.-M. Chem. Phys. Lett. 2005, 403, 343.

[13] Strano, M. S.; Dyke, C. A.; Usrey, M. L.; Barone, P. W.; Allen, M. J.; Shan, H.; Kittrell, C.; hauge, R. H.; Tour, J. M.; Smalley, R. E. Science 2003, 301, 1519.

[14] O’Connell, M. J.; Bachilo, S. M.; Huffman, C. B.; Moore, V. C.; Strano, M. S.; Haroz, E. H.; Rialon, K. L.; Boul, P. J.; Noon, W. H.; Kittrell, C.; Ma, J.; Hauge, R. H.; Weisman, R. B.; Smalley, R. E. Science 2002, 297, 593.

[15] Lee, J.-H.; Yoon, S.-M.; Kim, K. K.; Cha, I.-S.; Park Y. J.; Choi, J.-Y.; Lee, Y. H.; Paik, U. J. Phys. Chem. C 2008, $112,15267$.

[16] Moore, V. C.; Strano, M. S.; Haroz, E. H.; Hauge, R. H.; Smalley, R. E. Nano Lett. 2003, 3, 1379.

[17] Kim, K. K.; Bae, D. J.; Yang, C.-M; An, K. H.; Lee, J. Y.; Lee, Y. H. J. Nanosci. Nanotech. 2005, 5, 1055.

[18] Kim, K. K.; Yoon, S.-M.; Choi, J.-Y.; Lee, J.; Kim, B.-K.; Kim, J. M.; Lee, J.-H.; Paik, U.; Park, M. H.; Yang, C. W.; An, K. H.; Chung, Y.; Lee, Y. H. Adv. Func. Mater. 2007, 17, 1775.

[19] Jeong, M. S.; Byeon, C. C.; Cha, O. H.; Jeong, H.; Han, J. H.; Choi. Y. C.; An, K. H.; Oh, K. H.; Kim, K. K.; Lee, Y. H. NANO 2008, 3, 101.

[20] Ausman, K. D.; Piner, R.; Lourie, O.; Ruoff, R. S.; Korobov, M. J. Phys. Chem. B 2000, 104, 8911.

[21] Jeong, S. H.; Kim, K. K.; Jeong; S. J.; An, K. H.; Lee, S. H.; Lee, Y. H. Synth. Met. 2007, 157, 570.

[22] Geng, H. Z.; Lee, D. S.; Kim, K. K.; Kim, S. J.; Bae, J. J.; Lee. Y. H. J. Kor. Phys. Soc. 2008, 53, 979.

[23] An, K. H.; Lee. Y. H. NANO 2006, 1, 115. 